\title{
ENVEJECIMIENTO DE LA POBLACIÓN EN LA REGIÓN DE MURCIA: CAUSAS Y CONSECUENCIAS
}

\author{
Carlos Moya Ortega y Ramón García Marín ${ }^{2}$ \\ Universidad de Murcia
}

\section{RESUMEN}

Las importantes ganancias en esperanza de vida logradas en los últimos años, junto con tasas de fecundidad inferiores al nivel de reemplazo, han originado un progresivo envejecimiento de la población española, que se manifestará en un descenso ininterrumpido de la población en edad de trabajar y en un aumento continuado de la proporción de población de más de 65 años. La Región de Murcia, aunque presenta mejores expectativas demográficas que otras regiones españolas, también experimenta un notable envejecimiento de su población. De este modo, si no se mejora la situación actual de dependencia y recesión económica, el Estado del Bienestar puede verse comprometido en su mantenimiento y su continuidad será más difícil con una estructura demográfica envejecida.

Palabras clave: envejecimiento, Región de Murcia, dependencia, Estado del Bienestar.

\section{POPULATION AGING IN THE MURCIA REGION: CAUSES AND CONSEQUENCES}

\section{ABSTRACT}

The significant gains in life expectancy achieved in recent years, along with reduced fertility rates, below replacement level, have led to a gradual aging of the Spanish population, which will manifest itself in a continuous decline in the population of working age and a continued increase in the proportion of population older than 65 years. Murcia Region, though has better demographic expectations than other Spanish regions, also experiencing an acute population ageing. In this way, if the current situation of dependence and economic recession there is not improved, the Welfare State can be compromised in its maintenance and its continuity will be more difficult with an aged demographic structure.

Key words: ageing, Murcia Region (Spain), dependency, Welfare State.

\footnotetext{
Fecha de recepción: 4 de diciembre de 2014. Fecha de aceptación: 27 de febrero de 2015.

1 Graduado en Geografía (Universidad de Murcia), Masterando en la Universidad de Alicante. E-mail: moya.ortega.carlos@gmail.com

${ }^{2}$ Profesor Contratado Doctor, Departamento de Geografía, Universidad de Murcia, C/Santo Cristo s/n, 30001 Murcia (España). E-mail: ramongm@um.com
} 


\section{INTRODUCCIÓN}

La reducción de la tasa de natalidad, junto con una tasa de mortalidad casi estancada en las últimas décadas, ha ocasionado en España una desaceleración significativa en el ritmo de crecimiento de la población (REQUES, 2006). Recientemente, como consecuencia del vigoroso proceso inmigratorio (SERRANO MARTÍNEZ, 1999; GOZÁLVEZ PÉREZ, 2010), la reducción poblacional se ha visto paliada en numerosas comunidades autónomas. Asimismo, se ha producido un incremento significativo de la esperanza de vida al nacer. Estas variables han provocado una alteración de la estructura por edades de la población, ya que está aumentando la misma en edades más avanzadas y disminuyendo la población joven (EUROPEAN COMMISSION, 2014).

El envejecimiento de la población es un proceso inevitable e irreversible en las sociedades demográficamente avanzadas. Es una consecuencia natural de la transición demográfica, que alude al paso de un régimen demográfico de alta mortalidad y alta natalidad a otro de baja mortalidad y baja natalidad. El descenso de la mortalidad y de la fecundidad conlleva necesariamente un aumento progresivo del segmento de población mayor con respecto al segmento de población joven (CASTRO MARTÍN, 2010).

Dicho envejecimiento se entiende como un incremento sustancial y permanente del número de personas mayores de 64 años en relación con el resto de la población (PELÁEZ HERREROS, 2008), de modo que se modifica la estructura por edades de ésta (GÓMEZ FAYRÉN et al., 1994). Esta alteración se traduce en un descenso del porcentaje de la población en edad de trabajar o cotizantes y en un aumento de la población de más de 65 años o jubilados, lo que supone un incremento de la tasa de dependencia (HERNÁNDEZ DE COS y ORTEGA REGATO, 2002; ALONSO MESEGUER y CONDE-RUIZ, 2007). Esto conlleva una importante presión sobre la sostenibilidad de los sistemas de pensiones de reparto (CAIXA CATALUNYA, 2009), más aún si se tiene en cuenta la elevada tasa de paro que presenta la Región de Murcia, la cual se sitúa en séptimo lugar entre las comunidades autónomas españolas para el año 2014, con un 27,26\% (INE). Esta cifra es variable entre la población, ya que el paro se acentúa más en la población joven menor de 25 años $(58,42 \%)$ que en la adulta mayor de 25 años $(24,59 \%)$. Ello altera en parte la fecundidad de los jóvenes al tener menos oportunidades de empleo y emancipación.

Por otro lado, la natalidad y fecundidad se han visto paliadas en cierta medida por la llegada masiva de inmigrantes extranjeros, lo que ha ayudado a detener el proceso de envejecimiento global de la población (SERRANO MARTÍNEZ y GARCÍA MARÍN, 2011) y a atenuar desequilibrios en el mercado de trabajo (CASTRO MARTÍN, 2010).

Sin embargo, los efectos de la crisis económica y financiera sobre la inmigración se manifestaron a partir de finales del año 2008 con el aumento del desempleo (ALBA MONTESERÍN et al., 2013), lo que ocasiona un cambio de tendencia, pudiéndose aventurar el final de un ciclo demográfico y el inicio de otro, opuesto al anterior.

Si en las próximas décadas las tendencias de fecundidad y esperanza de vida se mantienen y cesan las llegadas de inmigrantes, continuando las salidas de población joven española hacia el exterior, el crecimiento de la población anciana seguirá incrementándose progresivamente. Sin embargo, dentro de este panorama, la Región de Murcia posee unas tasas de natalidad y fecundidad superiores a la media nacional y a las de la mayoría de las comunidades autónomas. Es decir, ha experimentado un envejecimiento demográfico, pero en menor medida que el conjunto español.

Si observamos la Figura 1 se puede contemplar cómo la Región de Murcia se sitúa en un cuarto puesto con respecto a las demás comunidades autónomas. En peor lugar encontramos a Asturias, Galicia y Castilla y León, con tasas de envejecimiento acusadas. Esto se debe a que en estas comunidades autónomas predominan las actividades primarias y los paisajes rurales, por lo que desde épocas anteriores se ha producido una intensa emigración en ellas (JIMÉNEZ BLASCO, 2008). 


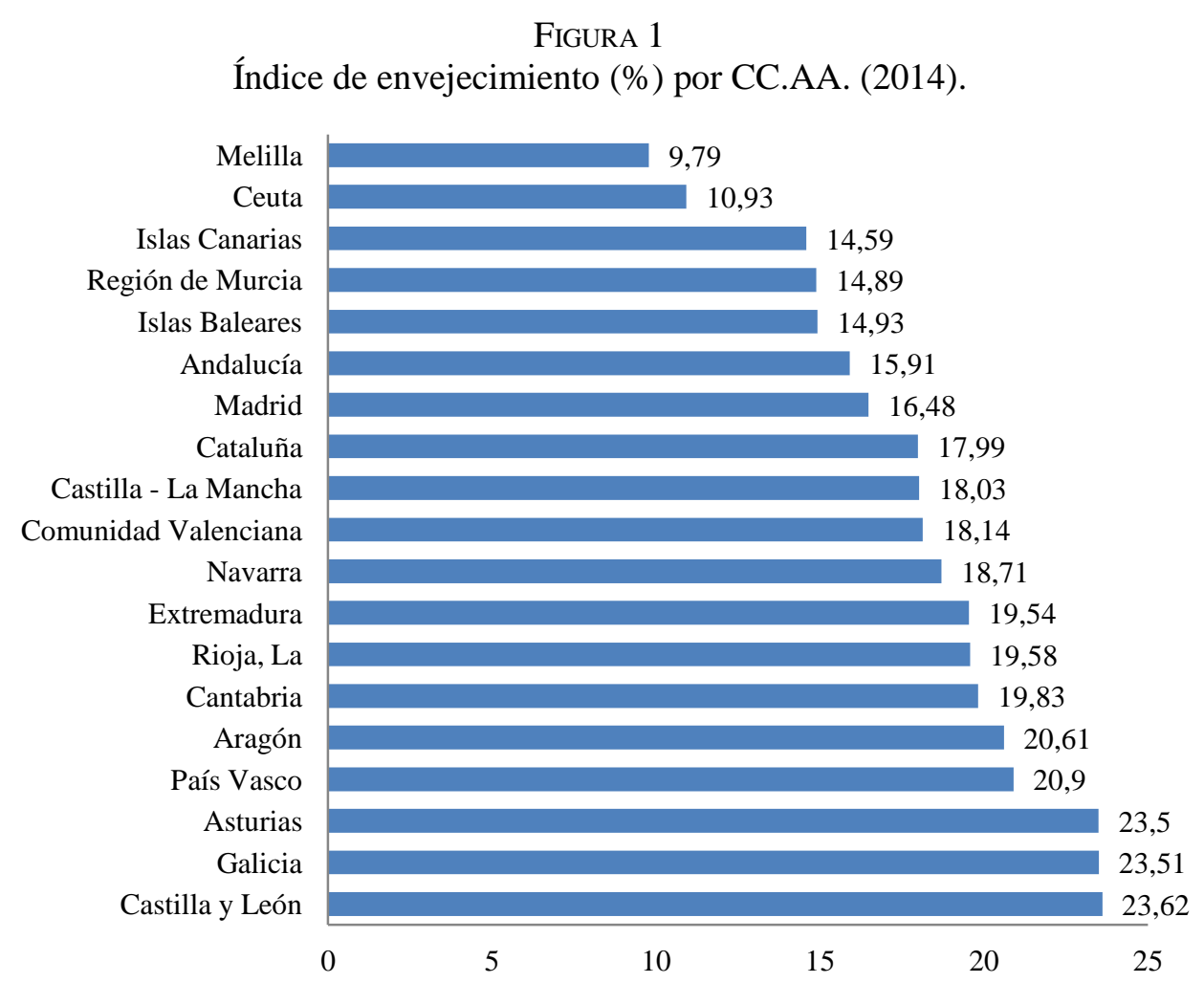

Fuente: Elaboración propia a partir de los datos extraídos del INE.

En la fachada mediterránea se superponen motivos de atracción que interesan a los extranjeros de todas las procedencias, donde destaca la benignidad que proporcionan el clima, el paisaje y la accesibilidad (GOZÁLVEZ PÉREZ, 2010). Estos factores, acompañados de la intensa urbanización, son los causantes de la atracción de población joven de diversas procedencias, lo que origina mayores tasas de fecundidad y natalidad y hace que la Región de Murcia posea mejores tasas demográficas. Sin embargo, estos factores también atraen a población envejecida, es decir, se produce una inmigración de jubilados atraídos en mayor medida por las condiciones climáticas tan favorables, que dan lugar a situaciones de ocio y tiempo libre. Este fenómeno se refleja con mayor asiduidad en los municipios del Sureste de la Región de Murcia, como Mazarrón, Cartagena y Los Alcázares. En consecuencia, la estructura por edades de estos municipios se ve alterada al aumentar la proporción de personas mayores de 65 años.

En este artículo se presenta la estructura y dinámica de la población de la Región de Murcia que, a pesar de exhibir datos más favorables que el conjunto español, está caracterizada en la actualidad por un proceso de envejecimiento demográfico.

El trabajo se organiza de la siguiente manera: en el epígrafe que sigue se expone la metodología seguida para el análisis de este proceso de envejecimiento. Posteriormente se estudia la estructura y dinámica de la población en la Región de Murcia durante la última década. A continuación se analizan tendencias espaciales del envejecimiento demográfico (análisis local), y finalmente, se concluye con un breve epígrafe de conclusiones y consideraciones finales, donde se expone una síntesis final de los distintos apartados y unas propuestas generales de necesaria actuación.

\section{METODOLOGÍA}

Para el estudio del envejecimiento poblacional en la Región de Murcia y sus diversos municipios se han tomado los datos de los censos de población y padrones municipales, sobre todo de la última década hasta la actualidad. Así, se ha comprobado la evolución de dicho 
envejecimiento. Además, el trabajo se ha centrado a nivel municipal, con el fin de conocer la incidencia del envejecimiento a escala local. La utilización de diversos índices ha sido el método escogido para establecer el grado de envejecimiento de la Región de Murcia y de los diferentes municipios, con el objetivo de determinar el lugar en que se encuentran con respecto a la media regional.

Los índices que se han calculado son el de envejecimiento ${ }^{3}$, sobreenvejecimiento $^{4}$ y dependencia para mayores de 64 años $^{5}$. Asimismo, se han utilizado otros datos y tasas, tales como la estructura de la población, fecundidad, natalidad, mortalidad y saldo migratorio. El empleo de numerosas fuentes de datos, en su mayoría procedentes del Instituto Nacional de Estadística (INE) y del Centro Regional de Estadística de Murcia (CREM), ha servido de apoyo para los análisis efectuados. Con los resultados obtenidos se han elaborado una serie de figuras que visualizan el fenómeno del envejecimiento, tanto para el conjunto de la población regional como para cada uno de los municipios.

\section{ESTRUCTURA Y DINÁMICA DE LA POBLACIÓN}

La Región de Murcia, al igual que las demás regiones del conjunto español, se ha caracterizado en los últimos años por poseer un movimiento natural de la población débil, determinado por una reducida tasa de natalidad y una tasa de mortalidad casi estancada, lo que da lugar a un mayor número de supervivientes con edades superiores a los 65 años y menos jóvenes. Este proceso se ha fortalecido por el alargamiento de la edad media de vida (CHASCO YRIGOYEN y HERNÁNDEZ ASENSIO, 2004; SERRANO MARTÍNEZ y GARCÍA MARÍN, 2013). Asimismo, este envejecimiento de la población también está ocasionando unas altas tasas de dependencia.

En las últimas décadas, la estructura por edades de la población murciana se ha alterado, cambiando su distribución; se ha acelerado el crecimiento progresivo de las personas mayores y ha disminuido la proporción de jóvenes (Tabla 1). Este proceso es similar en otras comunidades autónomas (PÉREZ DÍAZ y LECO BERROCAL, 2011; VIDAL DOMÍNGUEZ y FERNÁNDEZ PORTELA, 2014; RUBIERA, 2014).

La caída de la natalidad, fecundidad y mortalidad han ocasionado esta nueva estructura por edades, donde la población joven ha disminuido drásticamente, bajando 6,1 puntos porcentuales desde 1991 hasta 2014, mientras que la población anciana ha aumentado 4,66 puntos porcentuales durante el mismo periodo. De manera complementaria, el amplio grupo intermedio apenas modifica sus porcentajes. En cuanto a la esperanza de vida al nacimiento para los hombres y mujeres en la Región de Murcia se aprecia una ganancia de 5 años en tan sólo dos décadas. En 2013 los varones tienen una esperanza de vida cercana a 80 años y las mujeres cercana a 85 .

\footnotetext{
${ }^{3}$ Cociente entre personas de 65 años y más con respecto a la población total, multiplicado por 100.

${ }^{4}$ Cociente entre personas de 75 años y más con respecto a las personas entre 65-74, multiplicado por 100.

${ }^{5}$ Cociente entre personas de 65 años y más con respecto a las personas entre 16-64, multiplicado por 100.
} 
TABLA 1

Evolución de la estructura de la población residente en la Región de Murcia por grandes grupos de edad, para el periodo comprendido entre 1991-2014 (\%).

\begin{tabular}{lccc}
\hline & 0-14 años & 15-64 años & 65 y más años \\
\hline $\mathbf{1 9 9 1}$ & 23,81 & 66,10 & 10,09 \\
$\mathbf{1 9 9 6}$ & 19,97 & 68,31 & 11,72 \\
$\mathbf{1 9 9 8}$ & 19,04 & 68,82 & 12,14 \\
$\mathbf{1 9 9 9}$ & 18,67 & 68,89 & 12,44 \\
$\mathbf{2 0 0 0}$ & 17,66 & 67,87 & 14,47 \\
$\mathbf{2 0 0 1}$ & 17,19 & 68,48 & 14,33 \\
$\mathbf{2 0 0 2}$ & 17,11 & 68,65 & 14,24 \\
$\mathbf{2 0 0 3}$ & 17,15 & 68,74 & 14,10 \\
$\mathbf{2 0 0 4}$ & 17,19 & 68,72 & 14,09 \\
$\mathbf{2 0 0 5}$ & 17,05 & 69,20 & 13,75 \\
$\mathbf{2 0 0 6}$ & 17,05 & 69,16 & 13,80 \\
$\mathbf{2 0 0 7}$ & 17,12 & 69,13 & 13,75 \\
$\mathbf{2 0 0 8}$ & 17,18 & 69,22 & 13,61 \\
$\mathbf{2 0 0 9}$ & 17,36 & 68,99 & 13,65 \\
$\mathbf{2 0 1 0}$ & 17,55 & 68,68 & 13,76 \\
$\mathbf{2 0 1 1}$ & 17,62 & 68,32 & 14,05 \\
$\mathbf{2 0 1 2}$ & 17,63 & 68,07 & 14,30 \\
$\mathbf{2 0 1 3}$ & 17,66 & 67,81 & 14,53 \\
$\mathbf{2 0 1 4}$ & 17,71 & 67,54 & 14,75 \\
\hline
\end{tabular}

Fuente: Elaboración propia a partir de los datos extraídos del CREM. Padrón municipal de habitantes (varios años).

Estas diferencias por sexo se deben a la sobremortalidad masculina. Por un lado, la sobremortalidad masculina aparece con el predominio de las enfermedades degenerativas y la extensión de modos de vida y hábitos nocivos. Por otro lado, la esperanza de vida es mayor para las mujeres al poseer mayor fortaleza biológica por motivos hormonales e inmunológicos (LORENZO CARRASCOSA, 2004).

Desde finales de la transición demográfica ha tenido lugar una serie de cambios que han dado paso a una nueva realidad, complementario al aumento de la esperanza de vida. Las formas de vida tradicionales han ido perdiéndose, la mentalidad ha cambiado y los hijos no son apreciados del mismo modo que antes, ya que no son tan necesarios como lo fueron en el pasado. Este hecho ocasiona una pérdida significativa de la natalidad, reflejado en el retraso de la edad media de la maternidad.

La edad media de la maternidad ha pasado de los 29 años en 1991 a los 31 en 2013. Esto hace que el primer hijo sea más tardío y haya menos posibilidades de concebir otro, de manera que la fecundidad va disminuyendo conforme pasan los años (Figura 2). Desde 1991 hasta 2013, la fecundidad experimenta una alteración significativa, ya que por un lado reduce su tasa y por otro las mayores tasas han pasado de los 25-29 años hacia los 30-34. 
FIGURA 2

Evolución de la tasa de fecundidad según la edad de la madre en la Región de Murcia, por grandes grupos de edad, para el periodo comprendido entre 1991-2013 (\%).

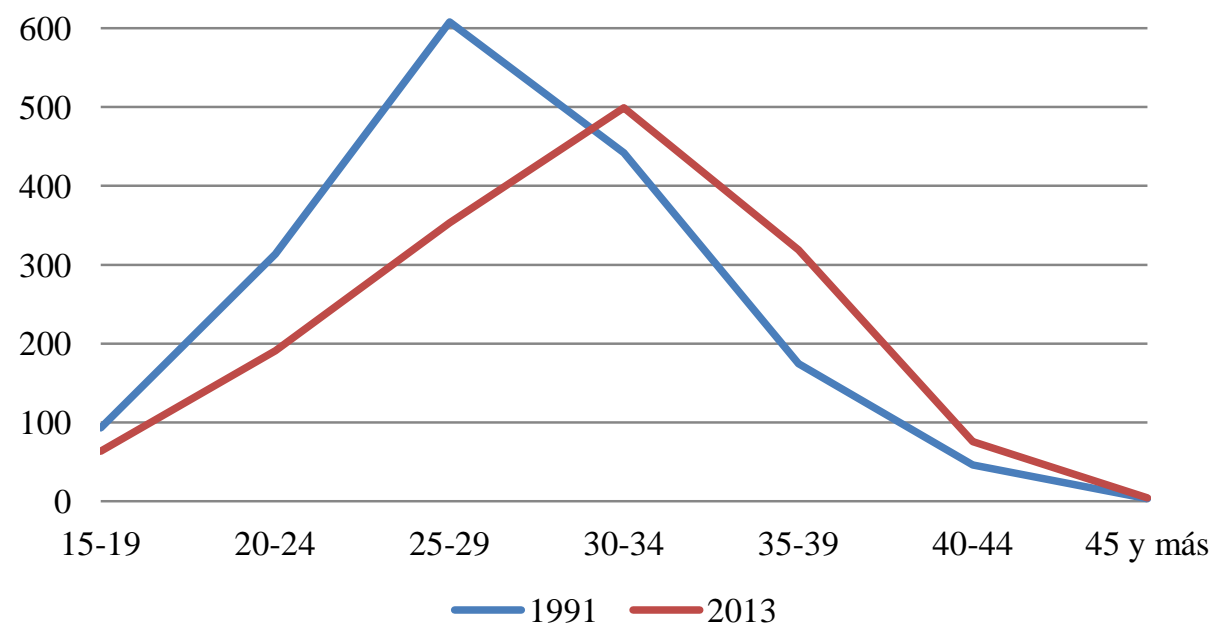

Fuente: Elaboración propia a partir de los datos extraídos del INE.

Esta disminución y evolución de las tasas de fecundidad se ha visto complementada, además, por la incorporación generalizada de la mujer al trabajo y el nuevo papel ascendente ocupado por ella en la sociedad. De este modo, las mujeres retrasan la maternidad hasta consolidar su situación laboral, por lo que la mayoría de los nacimientos se producen en el grupo de los 30-34 años (DELGADO et al., 2009; LEGAZPE MORALEJA, 2011). Esta nueva realidad en las formas de vida femeninas ayuda a entender las nuevas relaciones familiares, que cambian por completo los modelos y costumbres que eran característicos de épocas no muy lejanas (SÁNCHEZ FERNÁNDEZ y SÁNCHEZ MALDONADO, 2008).

Del mismo modo, el mercado de trabajo tampoco ayuda a consolidar una unidad familiar amplia, ya que la dinámica "paro-contratos temporales-paro" no anima a tomar un compromiso para toda la vida como es un hijo (CRESPO GARRIDO, 2013). Tampoco ayudan las dificultades para conciliar la vida familiar y laboral, debido a la escasez de guarderías a precios asequibles y el mantenimiento de comportamientos sexistas en el reparto de tareas domésticas y en el cuidado de los hijos. Por tanto, la familia numerosa, característica en el pasado, constituye en la actualidad una opción minoritaria (MONTORO, 2013). La sociedad ha desarrollado pautas de comportamiento poco favorecedoras para las familias numerosas, entre ellas el abandono de la población rural y su concentración masiva en las ciudades, que trae consigo una modificación en las formas de vida tradicionales.

Cabe resaltar la crisis económica acontecida en España a partir de 2008 como una de las causas que peores consecuencias ha tenido para la demografía española. Debido a ella se ha dado una menor intensidad en la nupcialidad, como causa del desempleo de numerosos jóvenes y las dificultades de acceso a la vivienda, lo que da lugar a que éstos se retrasen en salir de los hogares familiares. Consecuentemente, tardan más en casarse y así se produce un retraso en la edad del matrimonio y en la edad media para tener hijos, así como un acortamiento de las relaciones durante el periodo fértil de la mujer.

Estas circunstancias dan lugar a un número de hijos por mujer muy bajo, alejado del reemplazo generacional (Figura 3). La tasa de reposición de 2,1 hijos por mujer no se ve superada en ningún momento en el periodo comprendido entre 1991 y 2013. Es más, ni siquiera se acerca de manera suficiente a la tasa de reposición, ya que la tasa máxima a la que se llega en ese periodo es de 1,68 en el año 2008. 
FIGURA 3

Número medio de hijos por mujer en la Región de Murcia y para el total nacional (1991-2013).

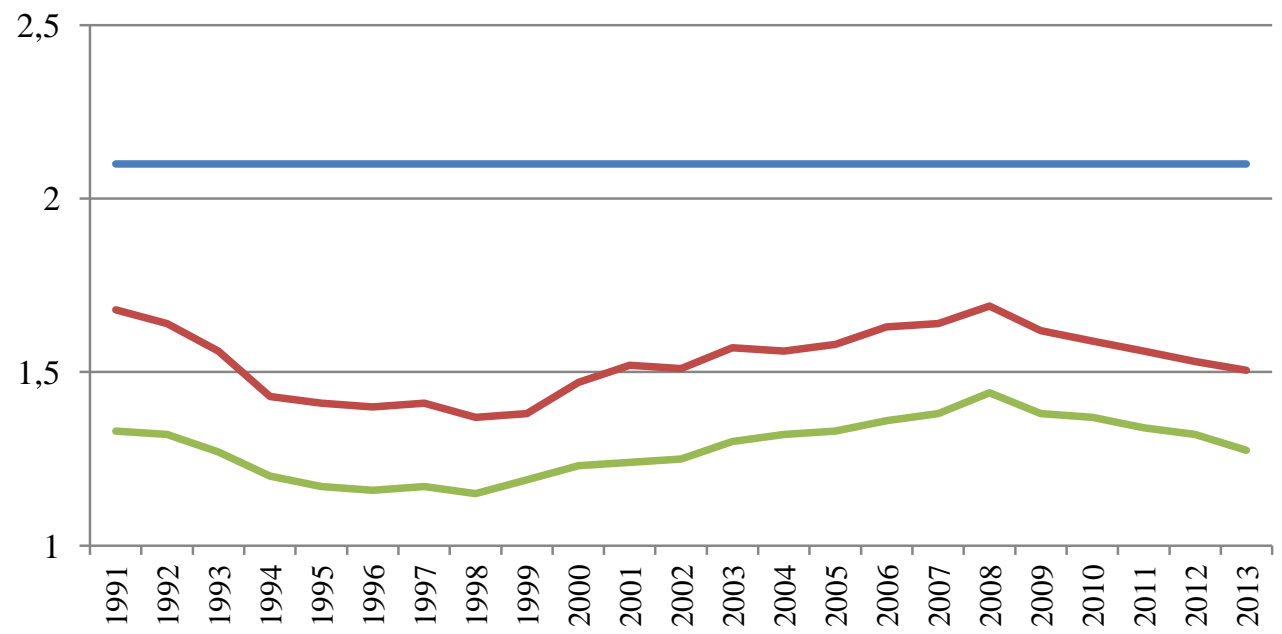

—Reposición —Región de Murcia Total Nacional

Fuente: Elaboración propia a partir de los datos extraídos del CREM.

El aumento progresivo del envejecimiento está causando una nueva realidad: el sobreenvejecimiento poblacional. El porcentaje de personas que superan los 75 años de edad está aumentando notablemente. Este proceso acentuado de envejecimiento no es muy beneficioso si se tiene en cuenta que esta población constituye el grueso del colectivo en el que se aplica el gasto de las cotizaciones. Las perspectivas para los próximos años acentúan la progresión de este importante colectivo y, con ello, la presión sobre los recursos privados y públicos para atender sus necesidades (CAIXA CATALUNYA, 2009).

La evolución de las variables demográficas descritas ha hecho que se modifique la forma de la pirámide de población (Figuras 4 y 5).

FIGURA 4

Pirámide de población de la Región de Murcia (1981).

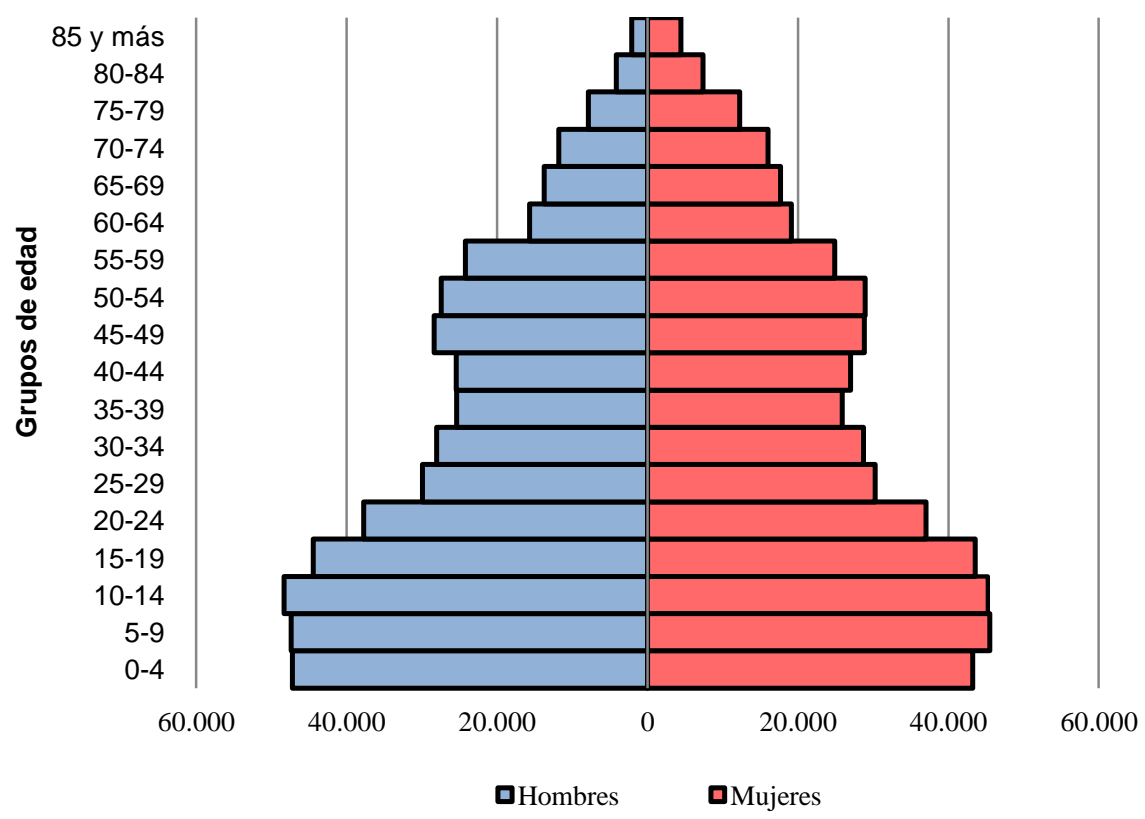

Fuente: Elaboración propia a partir de los datos extraídos del CREM. 
FIGURA 5

Pirámide de población de la Región de Murcia (2014).

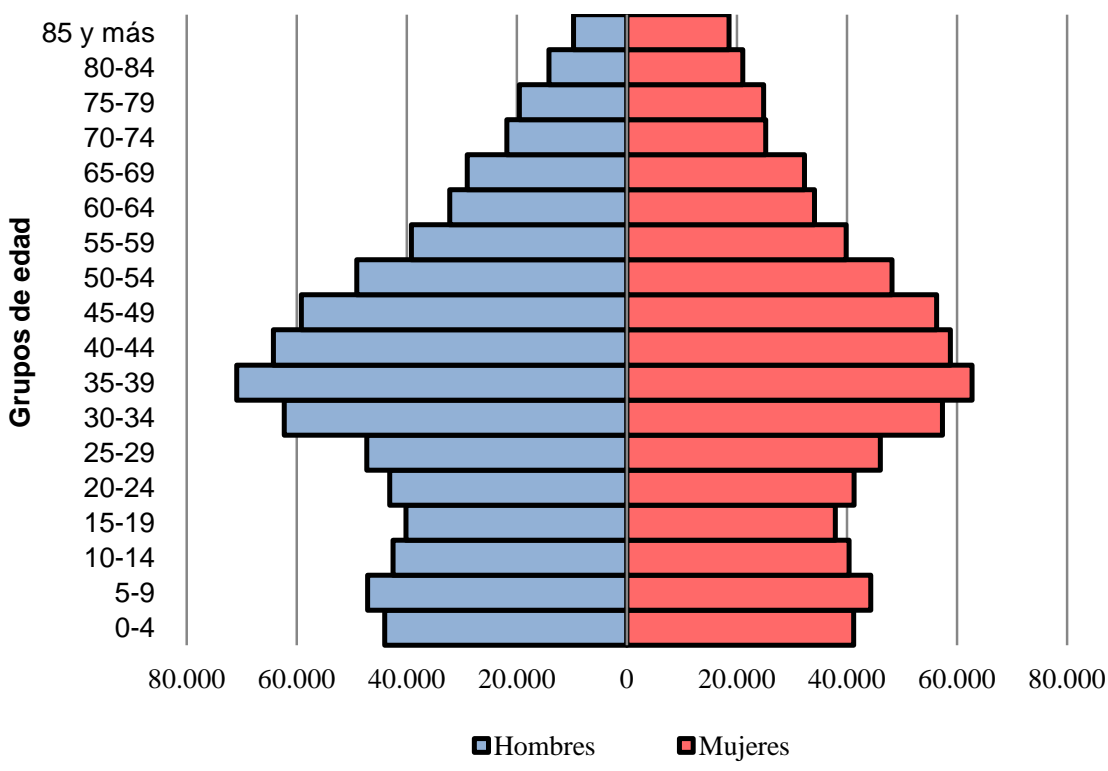

Fuente: Elaboración propia a partir de los datos extraídos del CREM.

De esta evolución de las pirámides de población de la Región de Murcia se desprende un detalle significativo: la tasa de envejecimiento demográfico cuenta con varios sumandos para crecer de modo acusado y sostenido, sobre todo a partir de 2020. Las causas son las siguientes: a) por falta de población debido a la débil natalidad actual; b) por la alta natalidad registrada en España durante el periodo 1955-1976 (baby boom), que llegarán a la edad de jubilación a partir de 2020; c) por la incorporación masiva de población extranjera a las ensanchadas cohortes correspondientes entre los 20 y 45 años; y d) por el progresivo aumento de la esperanza de vida (GOZÁLVEZ PÉREZ, 2010). De la misma manera se está perdiendo población en las franjas de edad más productivas (RUBIERA, 2014). Por lo tanto, nos queda una pirámide de población con la base estrecha, con una parte central engrosada y una cúspide en progresivo aumento (PÉREZ DÍAZ y LECO BERROCAL, 2011).

Las pirámides de población muestran cómo se ha producido un importante aumento de la población inmigrante, que ensancha aún más los grupos de edad jóvenes-adultos, situados entre los 25 y 39 años de edad. Estas cohortes se sitúan en condiciones ideales para aumentar la productividad económica y demográfica de España, aunque la crisis actual malogra estas excelentes posibilidades (GOZÁLVEZ PÉREZ, 2010). Por el contrario, los extranjeros tienen poca presencia en los grupos de más de 65 años, lo que indica que se trata de una inmigración de carácter económico en su mayor medida, mientras que tiene poco peso la inmigración de ocio y tiempo libre.

El engrosamiento de la pirámide de población en su parte central se ha visto favorecido por la intensa inmigración acontecida en España, pero de manera más destacada en la Región de Murcia (Figura 6), entre otras. Desde principios del siglo XXI hasta 2007, la comunidad murciana ha sido muy favorable en cuanto a la recepción de inmigrantes extranjeros (Figura 7).

La llegada de inmigrantes ha supuesto la aportación de nuevos individuos mediante dos vías: a través del propio flujo inmigratorio y mediante su reproducción biológica, lo que ha sido beneficioso no sólo para rejuvenecer la estructura demográfica de la población, sino también para sanear su debilitado crecimiento demográfico (MONLLOR DOMÍNGUEZ y GÓMEZ FAYRÉN, 2004).

En general los niveles de fecundidad de las mujeres extranjeras, en edades más tempranas, son bastante más elevados que los que acusan las mujeres españolas. La causa de este fenómeno hay que vincularla al desigual modo de concebir la familia que tienen estos dos colectivos 
poblacionales y a la importancia que alcanzan los hijos para el colectivo inmigrante, quienes los contemplan más como soporte y ayuda para la familia que como una carga (GÓMEZ FAYRÉN y MONLLOR DOMÍNGUEZ, 2005).

FIGURA 6

Pirámide de población de la Región de Murcia, según españoles y extranjeros (2014).

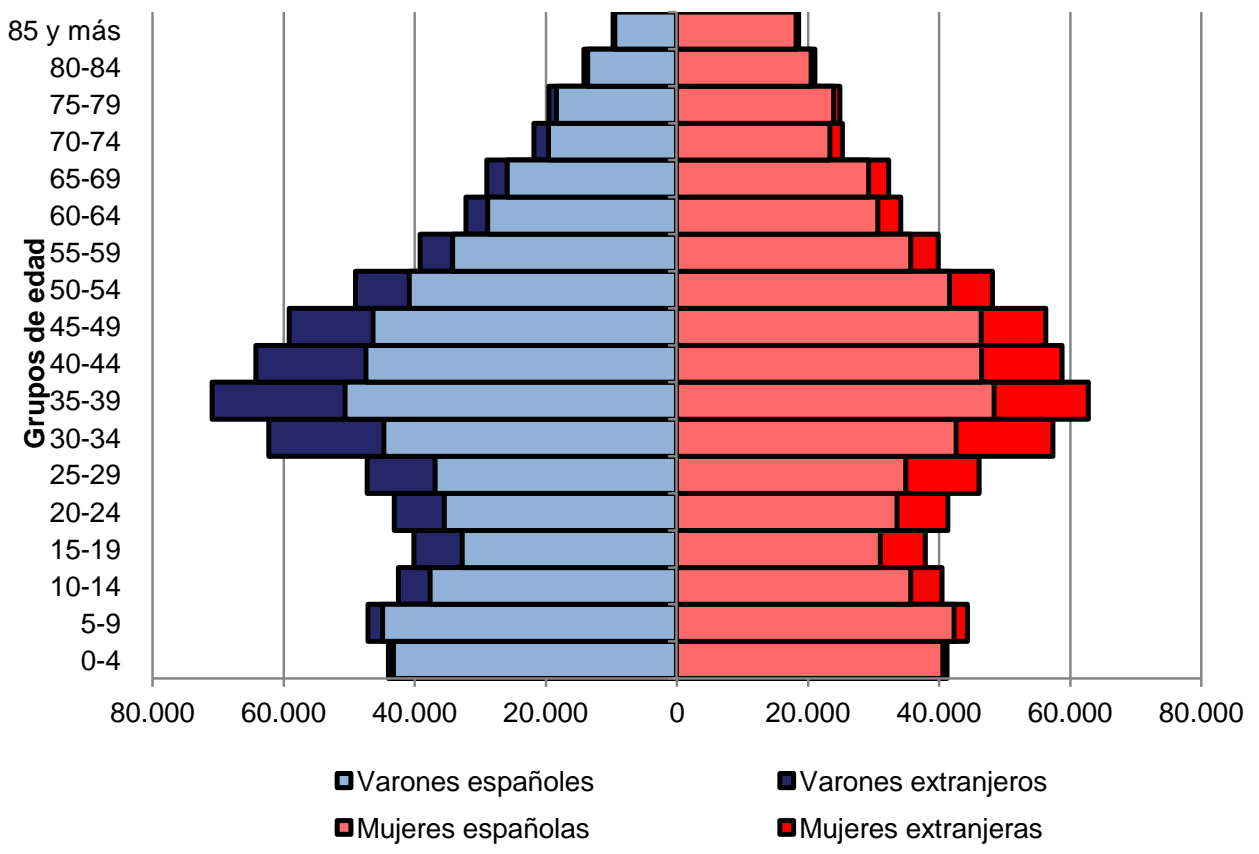

Fuente: Elaboración propia a partir de los datos extraídos del CREM.

FIGURA 7

Evolución de las inmigraciones procedentes del extranjero y las emigraciones al extranjero en la Región de Murcia (2002-2013).

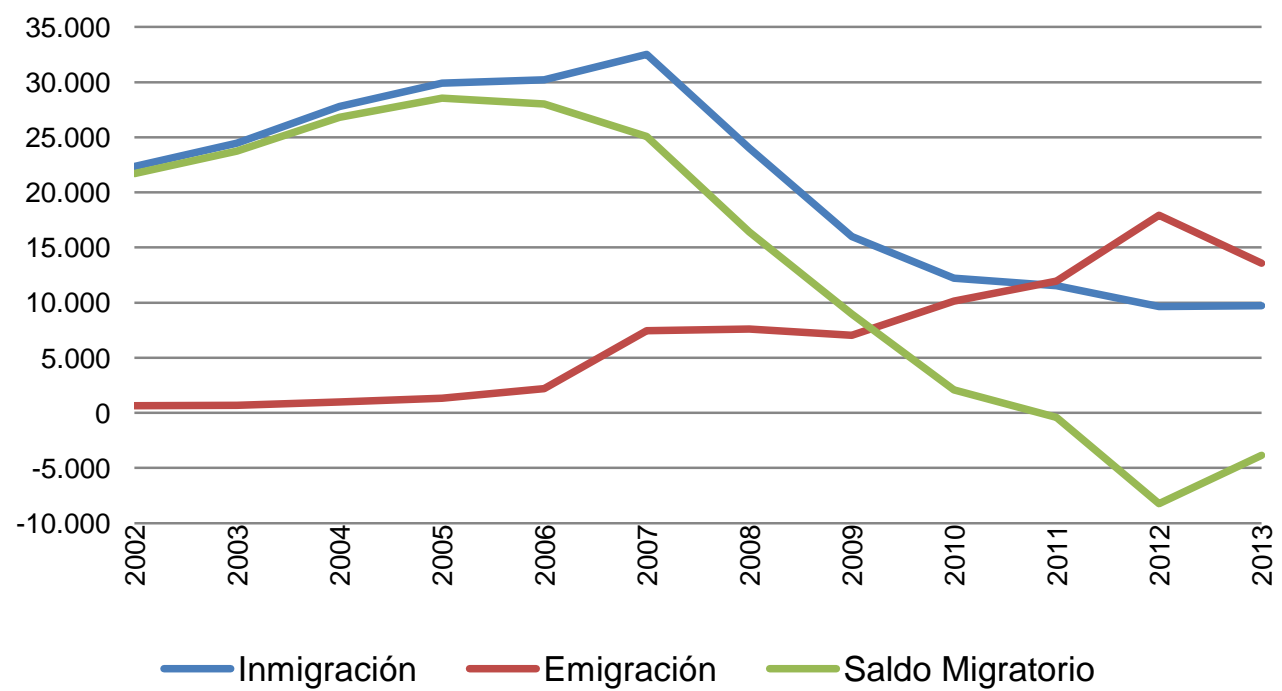

Fuente: Elaboración propia a partir de los datos extraídos del CREM.

Por edades, las mayores diferencias se sitúan entre los 15 y los 24 años (Figura 8). En estos grupos de edades los niveles de fecundidad de la mujer extranjera superan con creces a los de la mujer española, destacando el grupo de 15-19 años, donde el nivel de fecundidad de la mujer inmigrante es muy superior al de las españolas. De esa forma, también el número medio de hijos 
por mujer extranjera es mayor. Igualmente es superior al promedio nacional, que nunca supera los 2,1 hijos por mujer. Por ello, la proporción de hijos nacidos de madres extranjeras ha ganado un notable significado sobre el total de la población.

Pero, ¿cuál será la tendencia futura de las tasas de fecundidad de las mujeres inmigrantes? España no es un país en el que resulte beneficioso tener hijos, porque las ayudas son mínimas. Dado que los inmigrantes vienen a trabajar y pretenden ahorrar dinero o enviarlo a sus familias, no les conviene demasiado tener muchos hijos, pues ello minimiza sus posibilidades de trabajo y su capacidad de ahorro (JIMÉNEZ BLASCO, 2008).

FIGURA 8

Tasas de fecundidad por edad (\%o) según nacionalidad de la madre, españolas y extranjeras, para la Región de Murcia (2013).

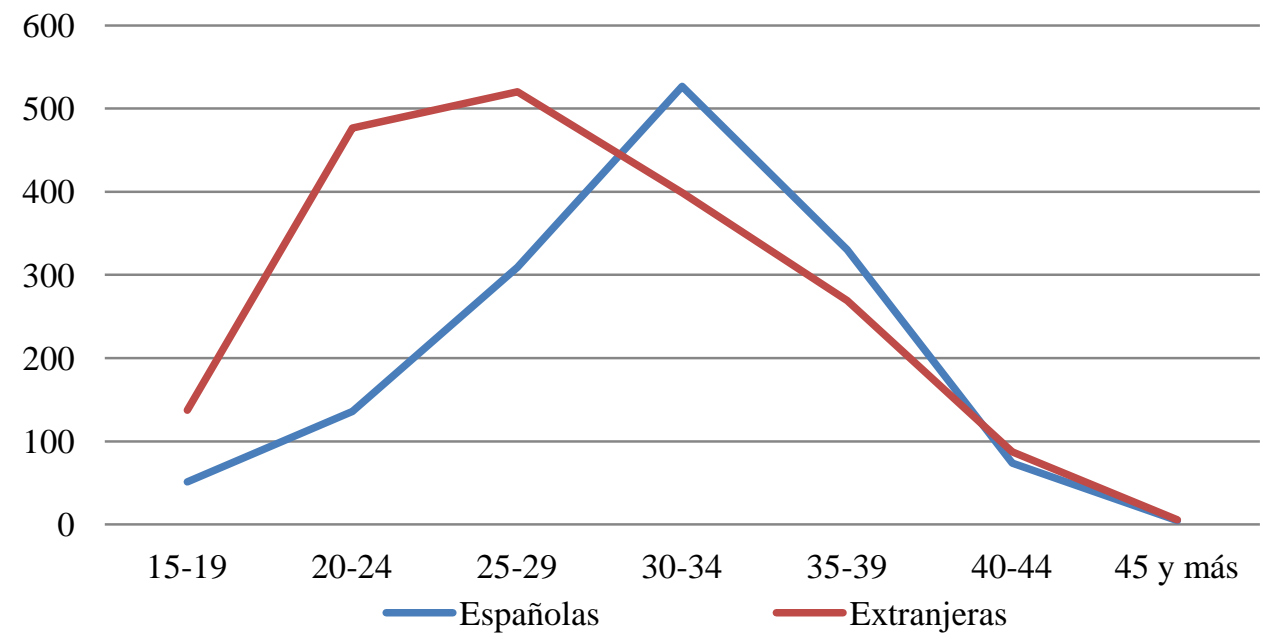

Fuente: Elaboración propia a partir de los datos extraídos del INE.

Puede decirse que la inmigración tiene un efecto rejuvenecedor transitorio, ya que los inmigrantes también envejecen (MACARRÓN, 2012). Por ello, no puede contrarrestar la tendencia al envejecimiento poblacional, sin embargo, sí puede retrasar levemente el proceso y mitigar temporalmente los desequilibrios entre población activa e inactiva. (CASTRO MARTÍN, 2010).

En la Figura 7 ha podido observarse el fuerte descenso de la población inmigrante, así como un incremento de los emigrantes, lo que origina un saldo migratorio negativo, algo que no sucedía hace décadas. La crisis económica desarrollada a partir de 2008 abre un nuevo escenario y en la actualidad nada favorece la llegada de nuevos inmigrantes extranjeros (SERRANO MARTÍNEZ, 2010); incluso están emigrando los ya asentados. Esto se debe a varios motivos. Uno es que buena parte de los inmigrantes sufre con más intensidad las consecuencias del desempleo, puesto que los trabajos que ha venido desempeñando la población inmigrante se han caracterizado por la temporalidad e inestabilidad. Otro motivo es que sólo una pequeña parte de los inmigrantes en situación de paro ha cotizado el tiempo suficiente para tener derecho a cobrar el subsidio de desempleo. Pero no sólo la población inmigrante asentada en España está emigrando, sino también la población española, por lo que los saldos migratorios extranjeros y españoles se vuelven negativos. Mientras que no se consiga un crecimiento económico saneado, vigoroso y duradero, dichos balances migratorios no podrán ser favorables (SERRANO MARTÍNEZ y GARCÍA MARÍN, 2013). 


\section{TENDENCIAS ESPACIALES DEL ENVEJECIMIENTO DEMOGRÁFICO EN LA REGIÓN DE MURCIA: ANÁLISIS LOCAL}

Puede afirmarse que existe una clara dicotomía entre el comportamiento que presentan los municipios de sierra con respecto a los litorales. Mientras que los primeros envejecen a un ritmo considerable y presentan en la actualidad un grado de envejecimiento alto con menor natalidad, en los municipios litorales el ritmo de envejecimiento es mucho menor y presentan mejores perspectivas demográficas. Esta situación responde a una serie de causas tanto demográficas como socioeconómicas.

En cuanto a las demográficas, se ha comprobado cómo las pautas de comportamiento seguidas por el ámbito litoral son distintas de las del ámbito serrano o de interior. El descenso de la natalidad y la fecundidad, la emigración de efectivos jóvenes y la escasa inmigración son los principales factores que explican ese acusado envejecimiento en los municipios interiores. Entre las causas socioeconómicas, destaca de forma preferente el desarrollo económico y territorial que presenta cada ámbito. La falta de oportunidades de empleo que los ámbitos serranos de interior ofertan a la población joven autóctona, así como los bajos niveles de renta y/o la primacía del sector agrario son algunos de los factores que explican las disparidades económicas y sociodemográficas existentes.

En el espacio regional, Gómez Fayrén et al. (1994) ya observaron una intensificación del envejecimiento hacia el interior y hacia el Noroeste, así como menos envejecimiento en los municipios con predominio de población urbana en relación a los rurales. El mapa del envejecimiento demográfico de la Región de Murcia para el año 2013 (Figura 9) muestra que hay 24 municipios por encima de la media regional (14,53\%). Esto indica que más de la mitad de los municipios de la Región de Murcia presentan tasas elevadas de envejecimiento.

FIGURA 9

Mapa de envejecimiento demográfico municipal, Región de Murcia (2013).

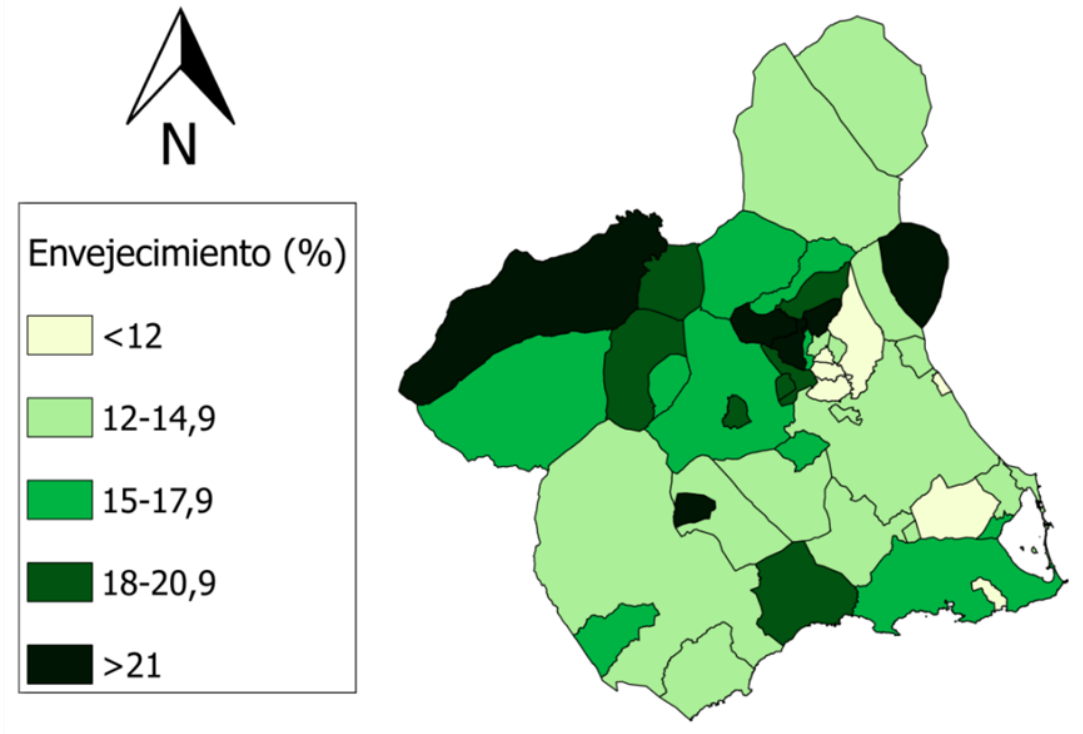

Fuente: Elaboración propia a partir de los datos extraídos del CREM.

Los municipios más envejecidos son los siguientes en orden ascendente: Ojós, Moratalla, Abanilla, Aledo, Ulea y Ricote. Estos territorios son zonas de despoblamiento rural con muy poca actividad económica y con dificultades de comunicación con los principales ejes vertebradores regionales, lo que ocasiona que no atraigan a población joven y que la existente emigre a otros municipios (o fuera de la Región de Murcia) en busca de nuevas expectativas para mejorar su nivel de vida. Este hecho origina un descenso de la natalidad y un aumento considerable de la mortalidad al encontrarse sólo población envejecida en dichos municipios. 
Algunos de los municipios situados por encima de la media presentan datos más favorables por su situación estratégica cerca de la capital regional (Lorquí, Santomera, Alguazas, Beniel, Ceutí, Las Torres de Cotillas y Molina de Segura), por lo que atraen a población hacia su área de influencia. Esto se debe a que el precio del suelo, el alquiler de viviendas y el precio de los servicios es mayor en Murcia que en estos municipios, y además cuentan también con abundantes servicios y una actividad económica dinámica.

Los municipios situados en el litoral de la Región presentan tasas también favorables, ya que atraen numerosa población joven. Se trata de municipios como Águilas, Cartagena, San Javier y San Pedro del Pinatar.

En cuanto al índice de sobreenvejecimiento en la Región de Murcia nos muestra una realidad parecida a la del índice de envejecimiento. Este índice evidencia el número de personas mayores de 74 años por cada 100 personas que se encuentran entre los 65 y los 74 . La media regional del índice de sobreenvejecimiento es de 98,8\%, lo que indica que 32 de los 45 municipios se sitúan por encima de la media.

La desaceleración en el ritmo de crecimiento de la población española ha estado acompañada de un aumento ininterrumpido de la ratio de dependencia (HERNÁNDEZ DE COS y ORTEGA REGATO, 2002), definida como la relación que existe entre la población que puede acceder a la pensión de jubilación (los mayores de 65 años) y la población en edad de trabajar (los comprendidos entre los 16 y los 64 años).

Este envejecimiento demográfico, que provoca las elevadas tasas de dependencia, influye también de manera directa en el sistema de pensiones, ya que se incrementa la ratio entre el número de pensiones y la población en edad de trabajar. Esto se traduce en un aumento del coste del sistema público de pensiones, ya que el esfuerzo económico necesario para mantener la pensión de los jubilados a un determinado nivel aumenta también (LÓPEZ DÍAZ, 2006; ZUBIRI, 2009). El crecimiento del número de personas mayores puede elevar las tasas de dependencia aumentando la presión sobre la población activa, ya que a mayor tasa de dependencia mayor será el número de personas que cada individuo económicamente activo debe mantener (GÓMEZ FAYRÉN et al., 1995). Mayores valores de la tasa de dependencia determinarían mayores niveles de gasto en pensiones por unidad de PIB, que pueden terminar afectando a la solvencia de un sistema de pensiones de reparto como el español (JIMÉNEZRIDRUEJO, 2008).

El aumento de la tasa de dependencia de los jóvenes se ha debido al retraso hasta los 16 años de la edad mínima legal para trabajar y a la prolongación de la escolaridad obligatoria, mientras que el aumento de la tasa de dependencia de los ancianos ha estado causada por la generalización de la jubilación pagada y por las prejubilaciones, que adelantan la salida del mundo laboral. Sólo mediante elevadas tasas de productividad es posible mantener las altas tasas de dependencia. No obstante, esto es muy difícil de llevar a cabo en la actualidad, debido a la situación de crisis económica.

Para finalizar este apartado cabe mencionar la relación existente entre el envejecimiento de la población y la inmigración. En la Figura 10 se observa perfectamente esta relación, ya que los municipios con mayor porcentaje de población extranjera poseen menor envejecimiento demográfico. En este sentido, puede decirse que la inmigración rejuvenece en cierta medida la población residente. Por un lado, aportando población en edades jóvenes y adultas, y por otro, contribuyendo con unas tasas de fecundidad superiores a las de la Región de Murcia. Aunque esto sucede de manera temporal, resulta beneficioso para paliar la debilidad demográfica existente. 
FIGURA 10

Proceso de inmigración en relación con el envejecimiento (2013).

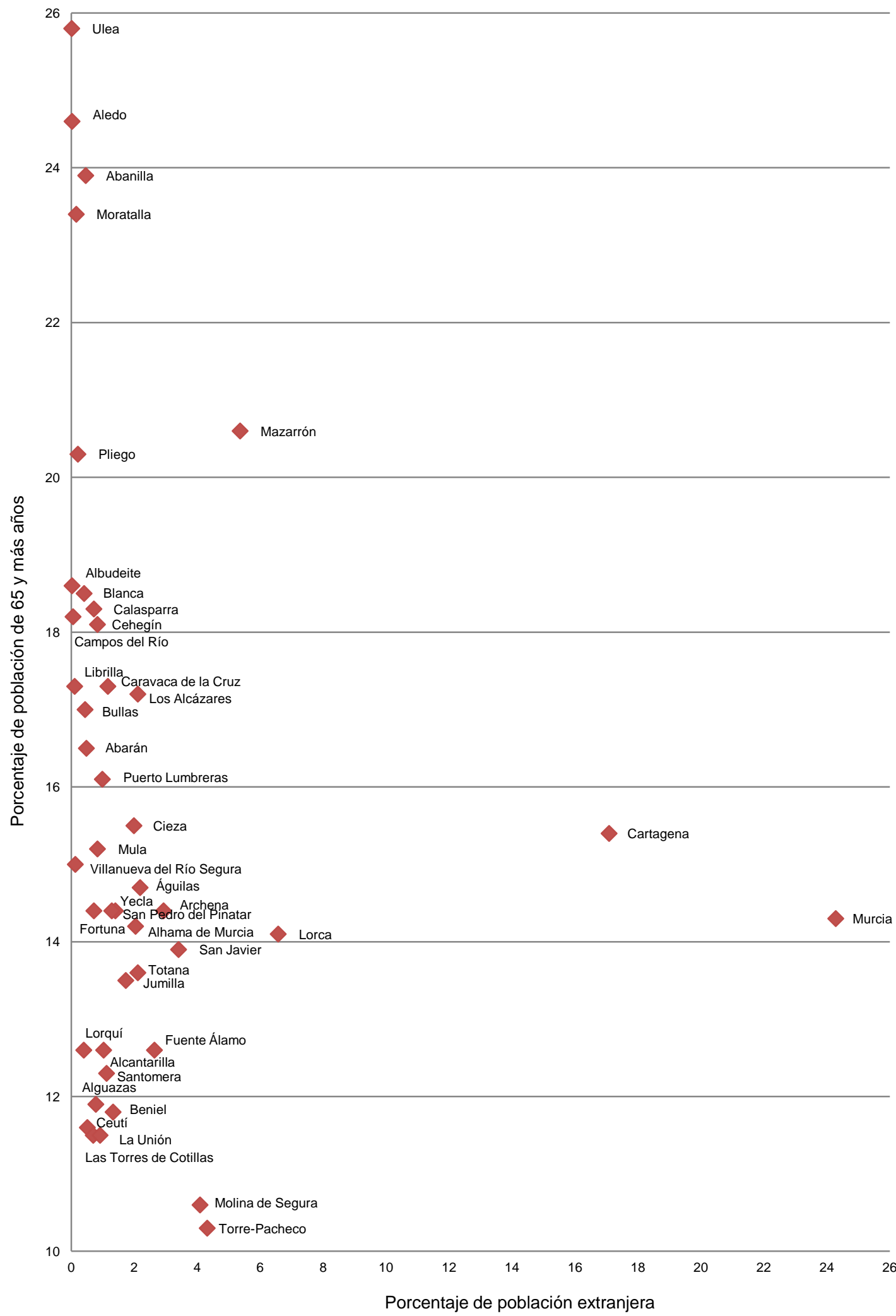

Fuente: Elaboración propia a partir de los datos extraídos del CREM. 


\section{CONSIDERACIONES FINALES}

La reducción de la tasa de natalidad y el incremento de la esperanza de vida provocará a largo plazo un progresivo envejecimiento de la población en la Región de Murcia, que se manifestará en un descenso ininterrumpido de la población en edad de trabajar y en un aumento continuado de la proporción de población de más de 65 años. Todo el sistema productivo se verá marcado por el devenir demográfico. Menores activos deberán ocuparse de mayores cohortes de población envejecida, en aumento constante. Asimismo, cuando las generaciones del baby boom se jubilen (sobre el año 2020), las consecuencias se agravarán, aumentándose la tasa de dependencia.

El incremento de la tasa de natalidad o la inmigración podría atenuar la tendencia al envejecimiento, pero no modificar sustancialmente la evolución de la tasa de dependencia. Sin embargo, ante la realidad de la crisis económica, parece muy poco probable que estos sucesos tengan lugar, ya que no existen actualmente políticas para incentivar la natalidad y la inmigración. En este momento, España no es un país que se vea como una oportunidad para mejorar el nivel de vida, incluso está teniendo lugar un considerable proceso de emigración al extranjero.

La evolución de la fecundidad se vería condicionada por la mayor facilidad de acceso de los jóvenes al mercado laboral, que podría adelantar su emancipación y la edad del matrimonio, por la existencia de políticas efectivas de conciliación de la vida laboral y familiar y apoyo a las familias, como facilidades para acceder a la vivienda, empleo estable para los jóvenes y guarderías a precios asequibles, así como también por la igualdad en el desempeño de las tareas domésticas y familiares.

En cuanto a la inmigración, si no se modifican pronto las circunstancias económicas y productivas, es probable que aumente su ritmo de salidas al exterior, así como que no lleguen en cantidades copiosas a este país.

De este modo, si no se ponen en marcha políticas adecuadas para mejorar la situación actual de dependencia y recesión económica, el Estado del Bienestar puede verse comprometido en su mantenimiento y su continuidad será más difícil con una estructura demográfica envejecida (UNITED NATIONS, 2014). La finalidad de estas reformas debe ser clara y decidida, pues es una certeza que en los próximos años se atenderá a un número mucho mayor de jubilados y no está suficientemente claro que se produzca un aumento proporcional en el número de afiliados a la Seguridad Social (BELMONTE et al., 2009).

Las tasas de natalidad actuales son difíciles de aumentar, más aún en el panorama de crisis económica grave que se presenta en la actualidad. Pero es un error pensar que no resulta viable realizar políticas sociales para impulsar la natalidad cuando es el pilar básico de toda sociedad para poder mantener el sistema de la Seguridad Social. Por ello, en la Región de Murcia, y en general en España, se debería empezar a considerar la puesta en práctica de políticas natalistas si no se quiere llegar a unos niveles mayores de envejecimiento. De este modo, la tasa de fecundidad aumentaría, con lo que se conseguiría diluir el peso de la tercera edad, a la vez que se limitaría la dependencia de la inmigración como vía para mantener el crecimiento poblacional.

Las consecuencias económicas que el envejecimiento general de la sociedad implica podrían ser solventadas si aumentara el número de cotizantes a la Seguridad Social. Se debe considerar el incremento del número de cotizantes mediante una mejora de las políticas de conciliación de la vida laboral y familiar de las mujeres, una regularización del empleo de los inmigrantes en situación de ilegalidad, el retraso de la edad de jubilación en algunos casos y el cambio en las contrataciones de los jóvenes y personas mayores, para así contrarrestar el descenso de la población en edad de trabajar.

En cuanto a medidas de tipo económico, la maternidad no tendría que ser un obstáculo insalvable para poder ejercer una carrera profesional. Existen ayudas para el cuidado de hijos o de natalidad que podrían generalizarse en muchas empresas y serían un buen punto de apoyo. Atendiendo a las políticas sociales, se debería crear una concienciación en la sociedad en lo que 
se refiere a la valoración de constituir una familia y su posterior atención. Para ello es imprescindible una conciencia social en la que domine la racionalización de los horarios (CRESPO GARRIDO, 2013). Asimismo, se debería reducir el periodo formativo de los jóvenes, evitar las responsabilidades familiares que las mujeres soportan por tradición, o limitar las prejubilaciones y el despido de los trabajadores de mayor edad (MUÑOZ DE BUSTILLO, 2007).

Si estas reformas no tienen lugar en los próximos años, la demografía se verá alterada a largo plazo y será menos probable una recuperación de la natalidad y fecundidad, con lo que habrá que hacer reformas estructurales en las políticas económicas y sociales para atender a las pensiones y se verán perjudicados otros ámbitos (MACARRÓN, 2012).

Si esta realidad continúa o se prolonga, quedará una región y un país en general con un crecimiento vegetativo mínimo, con un envejecimiento y una tasa de dependencia en continuo aumento y una población con un saldo migratorio negativo. En un futuro próximo, estos aspectos señalados podrían generar una regresión demográfica profunda, que originaría de forma inevitable reformas comprometidas en cuanto al sistema de la Seguridad Social para poder atender las necesidades básicas de la población y poder mantener, si se puede, el Estado del Bienestar.

\section{BIBLIOGRAFÍA}

ALBA MONTESERÍN, S.; FERNÁNDEZ ASPERILLA, A.I. y MARTÍNEZ VEGA, U. (2013): Estudios de la Fundación. "Crisis económica y nuevo panorama migratorio español». 65, $58 \mathrm{pp}$.

ALONSO MESEGUER, J. y CONDE-RUIZ, J.I. (2007): Información Comercial Española. «Reforma de las pensiones: la experiencia internacional». 837, pp. 179-193.

BELMONTE, L.J.; CORRALES, A.J. y RUIZ, J. (2009): European Journal of Education and Psychology. «La sostenibilidad del sistema de pensiones en España». Vol. 2, 2, pp. 113-129.

CAIXA CATALUNYA (2009): «Informe sobre el consumo y la economía familiar». 53, 84 pp.

CASTRO MARTÍN, T. (2010): Análisis del Real Instituto Elcano. «¿Puede la inmigración frenar el envejecimiento de la población española?» 40, $11 \mathrm{pp}$.

CHASCO YRIGOYEN, C. y HERNÁNDEZ ASENSIO, I. (2004): Encuentros multidisciplinares. «Aspectos económicos y territoriales del envejecimiento en España». Vol. 6, 16, pp. 47-58.

CRESPO GARRIDO, M. (2010): CLM Economía. «Mujer, mercado de trabajo y fecundidad: la conciliación de trabajo y familia, el mayor hándicap para el liderazgo femenino». 17, Universidad de Alcalá de Henares, pp. 169-196.

DELGADO, M. et al. (2009): Instituto de la Mujer. «Fecundidad y Trayectoria Laboral de las Mujeres en España». Madrid, 411 p.

EUROPEAN COMMISSION (2014): Eurostat. «Population ageing in Europe: Facts, implications and policies». Luxembourg, $76 \mathrm{pp}$.

GÓMEZ FAYRÉN J. y MONLLOR DOMÍNGUEZ, C. (2005): Papeles de Geografía. «Impactos de la inmigración extranjera sobre la reciente dinámica natural de la población regional (II)». 41-42, pp. 123-141.

GÓMEZ FAYRÉN, J. et al. (1994): Papeles de Geografía. «Envejecimiento en la Región de Murcia (I)». 20, pp. 147-173.

GÓMEZ FAYRÉN, J. et al. (1995): Papeles de Geografía. «Envejecimiento en la Región de Murcia (II)». 22, pp. 105-121.

GOZÁLVEZ PÉREZ, V. (2010): Investigaciones Geográficas. «Los extranjeros residentes en España: su aportación a la demografía». 52, Universidad de Alicante, pp. 99-135.

HERNÁNDEZ DE COS, P. y ORTEGA REGATO, E. (2002): Revista valenciana de economía y hacienda. «Gasto público y envejecimiento de la población». 6, pp. 9-35.

JIMÉNEZ BLASCO, B.C. (2008): Documents d'anàlisi geogràfica. «Desigualdades territoriales en relación con el envejecimiento de la población española». 52, pp. 91-110. 
JIMÉNEZ-RIDRUEJO AYUSO, Z. (2008): Revista del Ministerio de Trabajo e Inmigración. «Envejecimiento e inmigración: Consecuencias sobre la sostenibilidad del sistema de pensiones en España». 74, pp. 359-378.

LEGAZPE MORALEJA, N. (2011): I Jornadas Doctorales de Castilla-La Mancha. «Fecundidad y participación laboral femenina: una estimación simultánea». Universidad de Castilla-La Mancha, $25 \mathrm{pp}$.

LÓPEZ DÍAZ, J. (2006): Temas para el debate. «Envejecimiento y pensiones». 139, pp. 29-32.

LORENZO CARRASCOSA, L. (2004): Instituto Nacional de Estadística «Consecuencias del envejecimiento de la población: el futuro de las pensiones». $20 \mathrm{pp}$.

MACARRÓN LARUMBE, A. (2012): «El declive demográfico europeo, ¿quién pagará nuestras pensiones?» Seminario organizado por la Fundación Concordia, 42 pp.

MONLLOR DOMÍNGUEZ, C. y GÓMEZ FAYRÉN, J. (2004): NIMBUS. «La natalidad y la fecundidad de la población inmigrante en España». 13-14, pp. 143-165.

MONTORO, C. (2013): Servicio de Publicaciones de la Universidad de Navarra. «Relaciones entre familia y trabajo: Un análisis de la realidad española a partir de la Encuesta 'La familia, recurso de la sociedad'». 17, Pamplona, pp. 125-155.

MUÑOZ DE BUSTILLO, R. (2007): Ministerio de trabajo y Asuntos Sociales. «Extensión de la vida laboral o inserción temprana de jóvenes. Alternativas al sistema de pensiones». Madrid, pp. 188-190.

PELÁEZ HERREROS, Ó. (2008): Principios: Estudios de Economía Política. «Evolución del gasto en pensiones contributivas en España bajo distintos escenarios demográficos (20072050)». 12, pp. 45-60.

PÉREZ DÍAZ, A. y LECO BERROCAL, F. (2011): Geographicalia. «Envejecimiento, estancamiento poblacional y perspectivas demográficas en Extremadura». 59-60, pp. 309321.

REQUES, P. (2006): «Geodemografía: Fundamentos conceptuales y metodológicos». Santander, Universidad de Cantabria, $310 \mathrm{pp}$.

RUBIERA, F. (2014): «Análisis de la situación y perspectivas demográficas del principado de Asturias y revisión de las políticas aplicadas en Europa para el diseño de una agenda demográfica». Oviedo, $56 \mathrm{pp}$.

SÁNCHEZ FERNÁNDEZ, J. y SÁNCHEZ MALDONADO, J. (2008): Estadística española. «Fecundidad y actividad económica de las mujeres en España». Vol. 50, 169, Universidad de Málaga, pp. 431-453.

SERRANO MARTÍNEZ, J.M. (1999): Immigrazione e territorio. «Spagna: dall'emigrazione all'immigrazione, Prospettive future e nuove sfide del 2000». Editorial Bolonia, pp. 43-73.

SERRANO MARTÍNEZ, J.M. (2010): Migraciones. «Dinámica de la población española: ¿Hacia qué horizonte en los flujos migratorios exteriores?». 28, pp. 11-53.

SERRANO MARTÍNEZ, J.M. y GARCÍA MARÍN, R. (2011): Boletín Económico de ICE. «Evolución de la población residente en España. Un proceso reciente dinámico, pero ¿con discontinuidad?». 3017, pp. 47-63.

SERRANO MARTÍNEZ, J.M. y GARCÍA MARÍN, R. (2013): Papeles de población. «Regresión demográfica en España: crisis económica y cambio de ciclo migratorio». Vol. 19, 76, pp. 89-128.

UNITED NATIONS (2014): Department of Economic and Social Affairs Population Division. «Concise Report on the World Population Situation in 2014». 38 pp.

VIDAL DOMÍNGUEZ, M.J. y FERNÁNDEZ PORTELA, J. (2014): XIV Congreso Nacional de Población. «Castilla León la comunidad más envejecida de España: Perspectiva actual». Sevilla, pp. 95-107.

ZUBIRI ORIA, I. (2009): Revista del Ministerio de Trabajo e Inmigración. «El Sistema de Pensiones Español ante el reto del envejecimiento». 1, pp. 31-57. 